\title{
VibroTac: An Ergonomic And Versatile Usable Vibrotactile Feedback Device
}

\author{
Simon Schätzle, Tobias Ende, Tilo Wüsthoff, Carsten Preusche
}

\begin{abstract}
This paper presents an ergonomic vibrotactile feedback device for the human arm. Due to the developed concept, the device can be used for a large spectrum of applications and a wide range of arm diameters since vibration segments are self-aligning to their intended positions. Furthermore, the device improves user convenience and movement capability as it is battery powered and controlled through a wireless communication interface.

Vibrotactile stimuli are used to give collision feedback or guidance information to the human arm when interacting with a Virtual Reality scenario. The usefulness of this device has been shown in a Virtual Reality automotive assembly verification and a telerobotic system.
\end{abstract}

\section{INTRODUCTION}

Vibrotactile feedback belongs to the category of tactile feedback and forms along together with kinaesthetic feedback the so-called haptic feedback. The term haptic itself is understood as the perception of mechanical, thermal and or pain sensation; it is the process of recognizing the environment through touch. The mechanical sensation can be divided into kinaesthetic (information about force, torques, movements, position and angles of muscles and joints) and tactile (mechanical interaction with skin) perception [1].

Haptic feedback is an important source of information in our daily life for example when grasping, manipulating objects and identifying their (surface-) properties. Today, a wide spectrum of applications from which a few are listed exemplary are already using haptic feedback:

- mobile phones: vibration alarm and touch user interface with tactile feedback [2]

- computer games: force feedback joysticks

- sensory substitution e.g. Braille [3]

- medical diagnostic and therapeutics [4]

- human-machine interfaces for telerobotics or interaction with Virtual Reality [5]

- navigation hints e.g. for pilots [6].

Vibrotactile feedback is generated with devices that apply vibration stimuli to the human skin. The use of vibrotactile feedback is versatile and useful to enhance the level of intuitive perception of and interaction with the environment.

At DLR, a bimanual Man-Machine-Interface (MMI) [5] is used to interact with Virtual Reality (VR) scenarios or to operate a telerobotic system in order to manipulate at inaccessible or dangerous places. Besides kinaesthetic feedback (force and torque feedback), additional vibrotactile feedback

All authors are with Institute of Robotics and Mechatronics, German Aerospace Center (DLR), Oberpfaffenhofen, Germany firstname. lastnameddlr.de is applied to enhance the level of immersion into the VR scenario and to improve the user's performance in successful task completion.

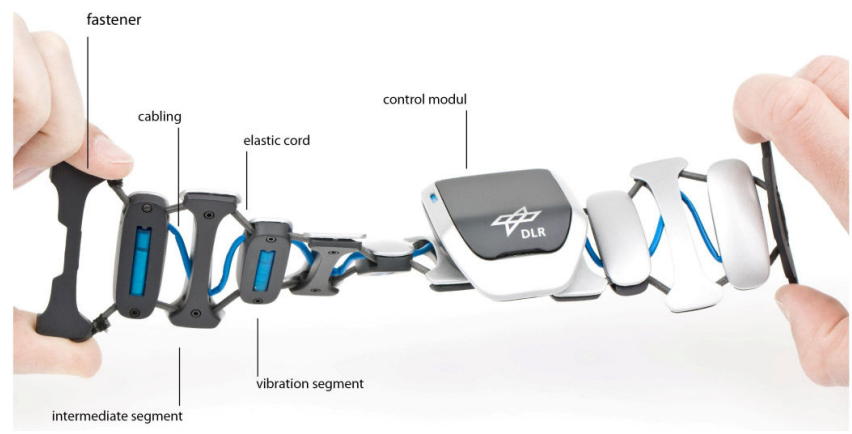

Fig. 1: The ergonomic vibrotactile feedback device

There are many research groups working on vibrotactile feedback and adequate devices [3], [6], [7], [8], [9]. Many prototypes have been built for use in laboratories. However, these devices were not designed with regard to ergonomic aspects. For example in [10], D. Tsetserukou and S. Tachi present a tactile feedback device for telemanipulation. A drawback of this device is a lack of automatic adaption to different arm diameters. Furthermore, this device has no fastener and needs to be slipped over like a bracelet. This drawback is discussed below.

DLR's VibroTac is a versatile usable, ergonomic and easyto-handle feedback device (Fig. 1) which will be introduced hereafter. The following section describes the main problems which have been faced. Section III introduces the electronic and communication features of the device. The design and ergonomic aspects of the hardware are discussed in section IV. Two use-cases and potential fields of application are described in section V, while section VI summarizes the main results and concludes with future work.

\section{PROBLEM DESCRIPTION}

In [11], we investigated the main factors influencing the basics of a vibrotactile feedback device for the human arm. Based on the results with regard to alignment, number and type of vibration motors two kinds of prototypes were built. On the one hand, using these vibrotactile feedback device prototypes in the above mentioned applications revealed a positive effect on immersion. Therefore, we have concentrated on vibrotactile feedback whereas other haptic modalities (e.g. voice coil or tactor actuators) have not been considered. On the other hand, the following shortcomings emerged: 
- Cables of power supply and communication compromise the user's movement capability.

- Different cuff sizes are necessary as they have a limited range of elasticity and arm diameters of different users vary considerable. This in turn implies an uncomfortable and unpractical use. Regardless of the different arm diameters, the vibration segments have to be positioned correctly (see IV A).

Consequently, two main improvements have been necessary:

- In order to avoid disturbing cables, battery powered electronics with wireless communication has been built as described in section III.

- A concept for an ergonomic design of the device has been developed and realized. Due to this concept the device can be used for a wide range of arm diameters since the vibration segments are self-aligning to their intended position. The development and design process is described in section IV.

\section{HARDWARE - ElECTRONICS AND ACTUATORS}

Due to wireless communication, battery power, a microcontroller and an ergonomic design, the user can easily adapt the position and number of feedback devices placed on the body's arms or legs depending on the needs of different applications. This section describes the electronic, communication and actuator features.

\section{A. Battery power}

Batteries are used in order to overcome the shortcoming of power cables which limit the user's movement capability. The performance of the battery with regard to capacity is a trade-off between size, weight and power. Each vibrotactile feedback device is powered by a Lithium-Ion battery with a rated voltage of $3,7 \mathrm{~V}$ and a capacity of $800 \mathrm{mAh}$. In order to determine the battery life, motors were activated sequentially one second at maximum intensity while battery voltage was recorded. The minimum operation duration is approximately 70 minutes (Fig. 2).

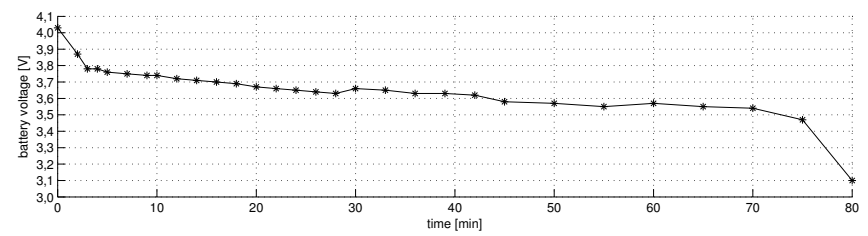

Fig. 2: Voltage-time diagram of Li-Ion battery with one motor activated at maximum intensity

To prevent damage to the battery, voltage monitoring switches the device off if the voltage falls below $2,9 \mathrm{~V}$ while discharging.

\section{B. Actuators}

In order to generate vibrotactile stimuli, standard cylindrical permanent magnetic DC vibration motors are used [11]. The vibration is generated by rotating an unbalanced mass at the end of the motor shaft. The motor and the rotating mass is encapsulated in a housing of $8.8 \mathrm{~mm}$ diameter. The operation voltage ranges from 0 to $4 \mathrm{VDC}$. At a rated voltage of $3 \mathrm{~V}$, the measured current is $250 \mathrm{~mA}$.

The vibration motors are continuously adjustable in frequency whereas the amplitude cannot be controlled separately. Since the perceived magnitude of the vibration depends mainly on the rotational speed of the mass, a frequency-voltage diagram is shown in Fig. 3. The maximum

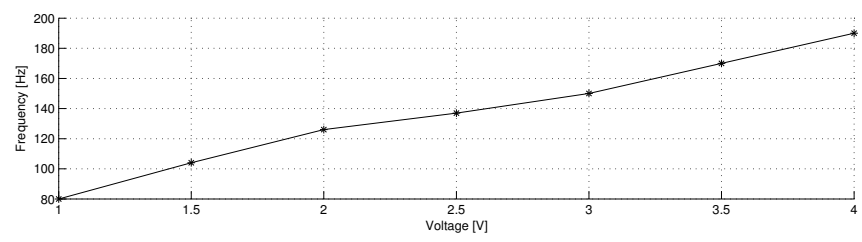

Fig. 3: Frequency-voltage diagram of a vibration motor

stimulation of skin mechanoreceptors occurs at a frequency of approximately $250 \mathrm{~Hz}$ [12]. This is in accordance with the results of Murray et al. [13]. Due to the frequency of approximately $180 \mathrm{~Hz}$ at the operating voltage, the vibration motors are well suited to generate vibrotactile stimuli for such a purpose.

\section{Wireless communication interface}

In contrast to disturbing data cables, the wireless communication interface ensures the user's movement capability. An integrated $\mathrm{XBee}{ }^{\circledR}$ radio frequency $(\mathrm{RF})$ module in the electronics passes the control commands to the microcontroller (Fig. 4). The RF-module is based on the IEEE 802.15.4 networking protocol and support network topologies like pointto-point, point-to-multipoint and peer-to-peer. They operate within the industrial, scientific and medical (ISM) $2.4 \mathrm{GHz}$ frequency band which is approved for use in Europe, the United States, Canada and Australia. The data rate of the RF communication is $250 \mathrm{kbit} / \mathrm{s}$. One RF-module is connected

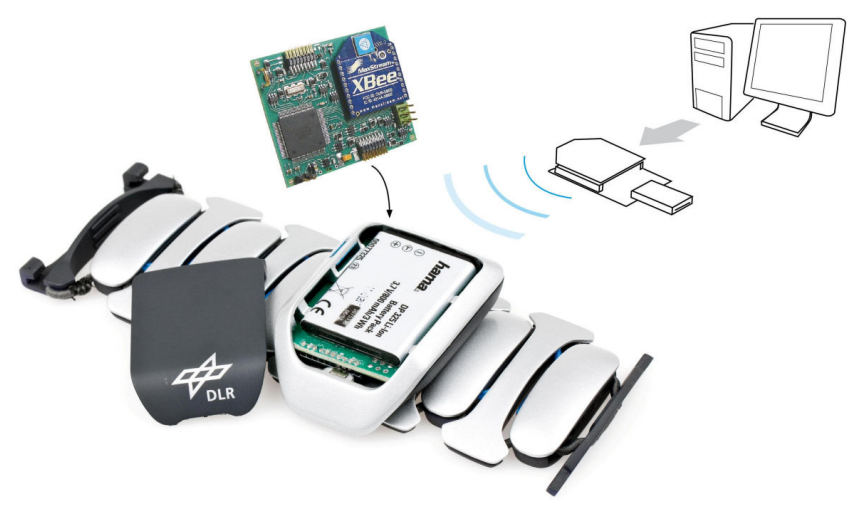

Fig. 4: Wireless control of VibroTac

to the control computer via universal serial bus (USB) and serves as master. Up to $64 \mathrm{RF}$-modules can be addressed separately from the master-module. Thus, each vibrotactile feedback device can be controlled completely independently within a range of at least $10 \mathrm{~m}$ inside buildings. 


\section{Control of vibration segments}

A microcontroller is used to activate motors and to control their intensity as well as the shape of stimulation. As an advantage compared to a hardwired solution, the firmware can be updated if changes of the device concerning control and user-interface are requested. A 8-bit microcontroller receives the commands from the RF-module with a maximum update rate of $1600 \mathrm{~Hz}$. If several devices are used, the update rate for each device is the maximum update rate divided through the number of devices. The microcontroller can independently control 15 vibration segments with a pulsewidth-modulation. As VibroTac has six vibration segments integrated, nine outputs are available for additional vibrotactile feedback such as finger feedback. A plug socket for fast cabling of external actuators is integrated in the device. Different kinds of tactile stimuli such as impulse, ramp, knock, continuous vibration and trembling can be generated.

\section{HARDWARE - DEVICE DESIGN AND ERGONOMICS}

During the development process of the device, ergonomic aspects have been carefully taken into consideration. Due to the developed concept, the device can be used for a wide range of arm diameters since vibration segments are selfaligning to their intended position. The following subsections describe the development process and its results.

\section{A. System requirements}

At the beginning of the development, several requirements have been defined:

1) Technical requirements

- six cylindrical vibration segments must be placed on the human arm's circumference in equal distances [11] and should have direct contact to the skin

- the cabling of the motors must guarantee a large elongation capability

2) Ergonomic requirements

- a large elongation capability of the device is required in order to facilitate wearability on wrist, forearm and upper arm

- the device must have a fastener and should not be slipped over the arm as other devices (e.g. finger feedback) worn on the hand may be used in combination

- the fastener of the device must be one-hand operable and must not interrupt the string of consistent stretch

- hygienic materials are required as several persons will be wearing the device.

\section{B. State of the Art}

There are already different solutions to attach items e.g. watches, music players or pockets to the human arm. These concepts are designed for use on a particular section of the arm. Generally they do not cover such a large elongation capability as claimed in the present case. For example, a watchband which is designed to be worn on the wrist can cover a very limited range of diameters only and cannot be worn on the upper arm. Furthermore, these solutions do not guarantee the correct positioning of several segments in equal distances on the circumferences of different arm diameters.

There are several research groups working on vibrotactile feedback and adequate devices. In [10] for example, D. Tsetserukou and S. Tachi present a tactile feedback device for telemanipulation. Its drawback is a lack of automatic adaption to different arm diameters thus requiring manual positioning of the segments and cables every time the device is worn on arms with different diameter. Furthermore, this device has to be slipped over like a bracelet as it has no fastener. This may cause problems if other devices are worn on the hand.

\section{Preliminary tests with mock-ups}

Several mock-ups were tested in order to validate function and intuitive handling of (existing) wristband-concepts, fastener mechanisms and material characteristics. Fig. 5 shows different concepts of fasteners which were tested with regard to one-hand operation and size. An example of different

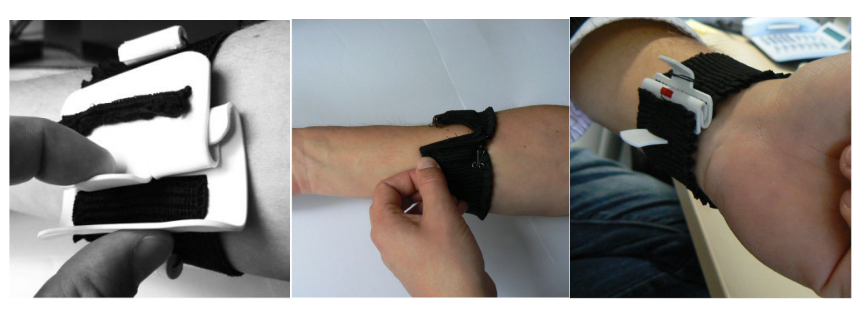

Fig. 5: Mock-ups of different fastener mechanisms

materials which were used are depicted in Fig. 6. Many products are made of flat and elastic materials such as rubber straps which are available in a rich assortment. Several tests were conducted to find a suitable material with regard to tensibility force and range of elastic lengthening. On the one

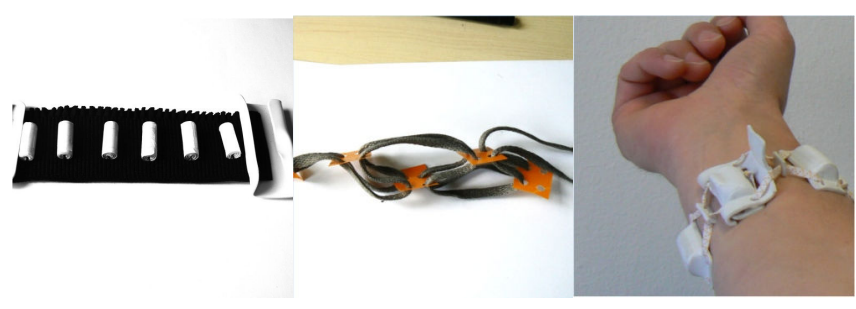

$\begin{array}{lll}\text { (a) flat materials } & \text { (b) nonelastic cord to tie } & \text { (c) elastic cord }\end{array}$

Fig. 6: Comparison of different material characteristics

hand, the material must reliably fix the vibration segments to the arm while on the other hand the material must be elastic enough to be used on larger arm diameters without being painful and constricting blood flow. As we could not find any material satisfying our requirements, we developed a new concept to obtain the desired features of the device. 


\section{Ergonomic concept and features of VibroTac}

In this subsection the concept which was developed to meet the claimed requirements is presented in detail. The description is divided into the following subsections: large range of elastic lengthening of the device, self-alignment of vibration segments, flexible cabling, one-hand operable fastener and hygiene.

1) Concept for large range of elastic lengthening: It is crucial to find a concept that enables the device to fit on a large range of arm diameters. The construction is based upon the data of ergonomic tables (Tab. I). This ensures that as many people as possible can use the device. A range from $15 \mathrm{~cm}$ to $38,5 \mathrm{~cm}$ of arm circumference covers the 5th percentile of the female wrist and upper arm. In order to

\begin{tabular}{|c|c|c|c|c|}
\hline age group $18-65$ years & \multicolumn{2}{|c|}{ male } & \multicolumn{2}{|c|}{ female } \\
\hline percentile & 5 & 95 & 5 & 95 \\
\hline wrist & $16 \mathrm{~cm}$ & $19 \mathrm{~cm}$ & $15 \mathrm{~cm}$ & $18 \mathrm{~cm}$ \\
\hline upper arm & $24,5 \mathrm{~cm}$ & $37,6 \mathrm{~cm}$ & $22,1 \mathrm{~cm}$ & $38,5 \mathrm{~cm}$ \\
\hline
\end{tabular}

TABLE I: Ergonomic table of wrist and arm circumferences [14]

obtain a large elongation capability, the vibration segments are linked together with elastic cords in a zigzag pattern (see Fig. 7 middle). Due to this pattern, the effective length of elastic material increases by about $35 \%$ in comparison to a straight connection of the segments (Fig. 7 bottom). The length of VibroTac in relaxed state is equal in both principles of segment linkage.

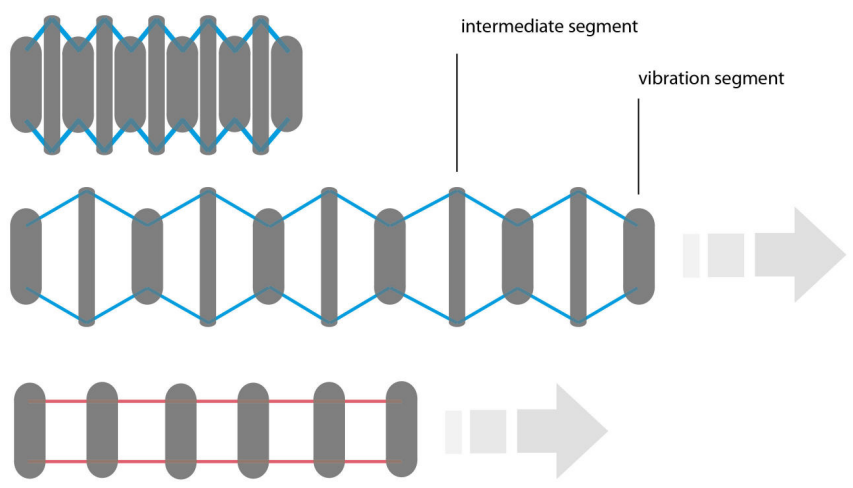

Fig. 7: VibroTac in relaxed state (top) and difference in elastic lengthening of zigzag geometry (middle) and straight geometry (bottom) if pulled with the same tensile force

Due to the slim segments, the device has a length of 15 $\mathrm{cm}$ in relaxed state and it reliably fits on a wrist with a circumference of $15 \mathrm{~cm}$ and larger (Fig. 8) as there is a distance of a few millimeters between the elastic cord and the skin due to the height of the segments which results in a slightly smaller diameter of the skin touching segments of the device.
Using elastic cords in zigzag pattern allows an elongation from $15 \mathrm{~cm}$ to $46 \mathrm{~cm}$ which satisfies the requirements of table I. Consequently the device fits comfortable on a male upper arm for example as depicted in Fig. 8 right.

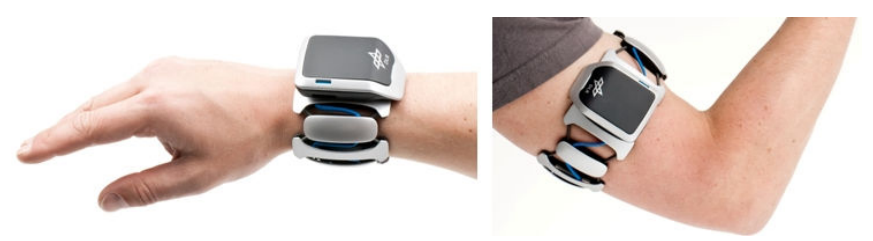

Fig. 8: Due to the large elongation capability, VibroTac can be worn on a the wrist (left) or even on the upper arm (right)

If both concepts of linking segments are compared, the difference in elastic lengthening becomes obvious. A construction with straight connection based on the same elastic cord and the same device length in relaxed state, stretches to $33 \mathrm{~cm}$ length only whereas the zigzag pattern reaches 46 $\mathrm{cm}$ (Fig. 7) if pulled with the same tensile force. As this lengthening is more than claimed in table $\mathrm{I}$, the device is more comfortable to wear as the tensile force is smaller than in the maximum stretched state. A maximum elasticity of $206 \%$ is reached for VibroTac, while the elastic cord itself has a elasticity of only $120 \%$.

Fig. 9 shows the spring constant $D=\frac{F}{\Delta L}$ of the zigzag pattern and the straight linking concept. In both cases, the same materials are used. The zigzag pattern has a positive

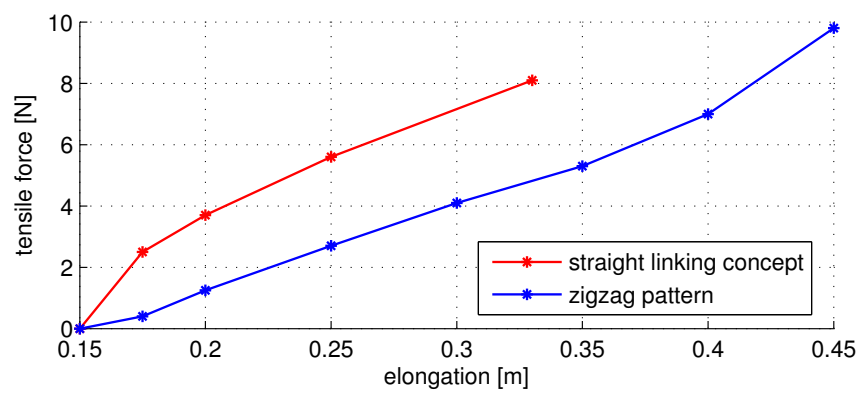

Fig. 9: Relation between tensile force and elongation

effect on the spring constant as $D_{\text {zigzag }}<D_{\text {straight }}$ which results in a more convenient use of the device without constricting blood flow.

2) Self-alignment of vibration segments: If the vibration segments are fixed on an inelastic strap in equal distances and the device is adapted to different arm diameters by changing the length of the strap with a velcro fastener for example, the vibration segments will be positioned in irregular distances with a resulting gap close to the fastener. In order to reach equal distances between the vibration segments on the arm's circumference both in the relaxed and in the stretched state, six elastic zones of the same length and elasticity are used between the vibration segments. Corresponding to the arm's diameter, all elastic zones are stretched similarly so that the motors are positioned with equal distances to each other (see Fig. 10). As the control module is mounted on top 
of one vibration segment, the regular distances between the segments are unaffected.

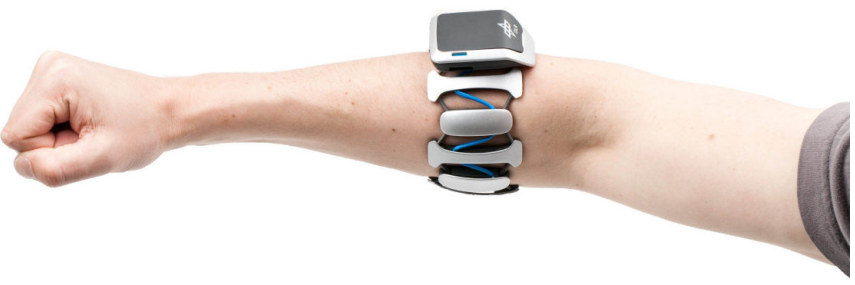

Fig. 10: VibroTac in stretched state with correct positioned segments

3) Flexible cabling: As the distance between the vibration segments is in correlation with the arm diameter, a cabling with high elongation capability and the ability to resist bending stress in numerous cycles is crucial. In this case a cable, which is used in textile products with integrated electronics, is applied. This highly elastic cable has a diameter of 0.66 $\mathrm{mm}$. Several cables are bundled in a heat shrinkable tube which connects the vibration segments as depicted in Fig. 11. The principle of meandering cables limits the bending radius of the cables and has a positive effect on their durability. The intermediate segments, which are used to create the zigzag pattern of the elastic cord, have a slot in which the cables are routed properly.

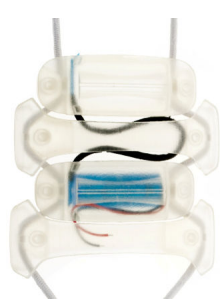

(a)

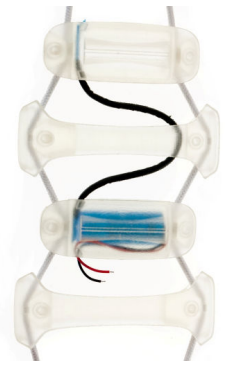

(b)
Fig. 11: Meandering cables in relaxed (a) and stretched (b) state

4) One-hand operable fastener: VibroTac is used in combination with other devices worn on the hand e.g. a dataglove or in combination with the coupling of the hand to a robot used as a haptic interface [5]. In order to simplify the handling of the device, it must have a fastener and should not be slipped over the arm. The fastener is designed in a way that two clamps can be connected with two fingers as depicted in Fig. 12. The fastener has roughly the same size as an intermediate segment. Thus it does not affect the equal distances between vibration segments.

5) Hygiene: As several persons use the device, hygienic aspects are relevant. Therefore it is important to avoid heating or sweat supporting constructions and materials. In contrast to flat materials covering large areas of the skin, the stiff segments and the elastic cords of VibroTac reduce perspiration. Furthermore, the components that are in contact with the skin can be dismounted, cleaned and disinfected.

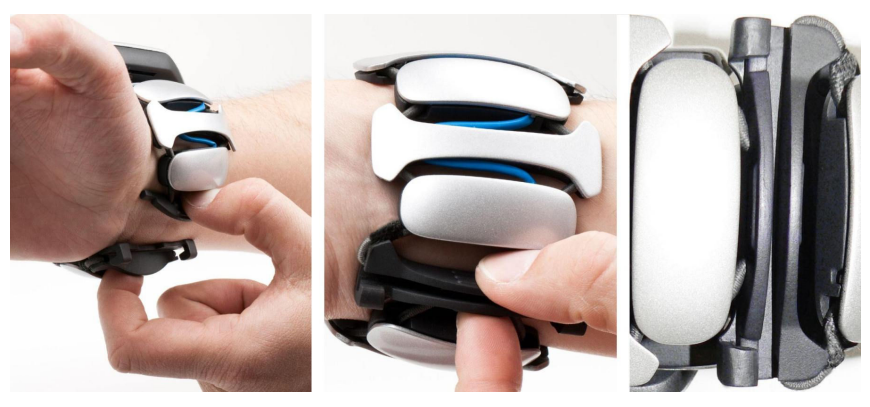

Fig. 12: One-hand operable fastener

\section{Applications and USE-CASEs}

\section{A. Collision feedback}

The immersion of virtual reality simulations is increased by introducing haptic feedback, i.e. rendering and displaying haptic information from the user's interaction with the virtual reality (VR) [1]. Haptic, or more precisely kinaesthetic displays like the PHANToM [15] or the DLR haptic interfaces [5] can present forces to a single point of interaction. Thus, the feedback of collisions occurring in the VR is limited to the hands. Here, VibroTac presents a solution to notify collisions between the human arm and objects within the VR. If the human arm collides with an object, a vibration stimulus is generated at the corresponding part of the arm.

At DLR, a bimanual Man-Machine-Interface is used for virtual assembly and maintenance verification (Fig. 13). Due to digital mock-ups within the VR scenario, engineers can evaluate the maintainability or mountability of mechanical parts within complex products such as cars or planes [16], [17]. Using VibroTac is an approach to intuitively verify the

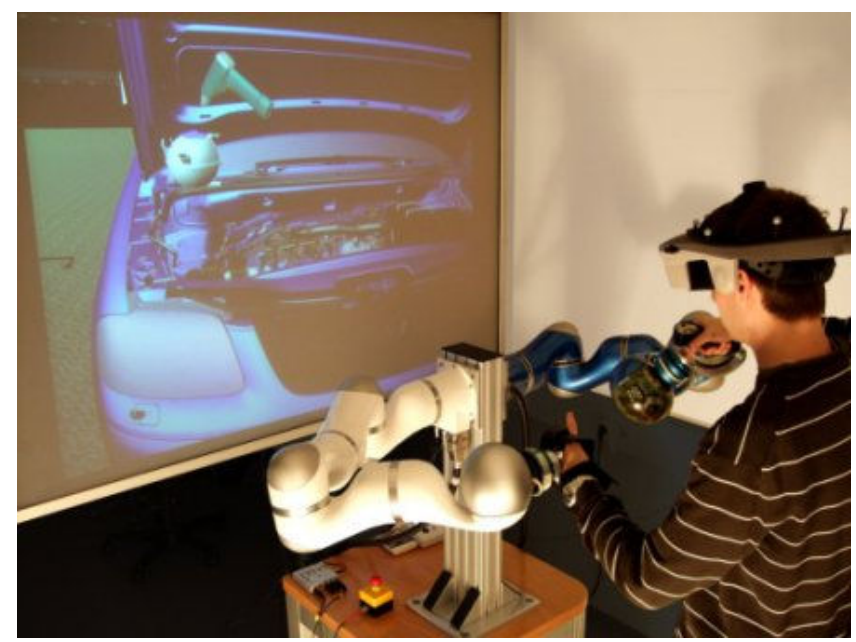

Fig. 13: A virtual assembly verification scenario

accessibility of the human arm to the workspace. This is of great interest besides the investigation whether the part itself is maintainable. Two exemplary kinds of stimuli in such an application are: $a$ ) increasing vibration intensity depending on the penetration depth into the virtual object or $b$ ) a single "knock" at the point of contact, eventually repeated as long as a the collision occurs. 


\section{B. Guidance}

Another field of application is the use of vibrotactile feedback to give guidance instructions e.g. translational or rotational movement hints for the arm. An example for this application is the transfer of skills in a remote maintenance scenario [19]. The vibrotactile feedback at the wrist of the apprentice or technician is controlled by a remote expert who can intuitively give guidance hints or notify (in-)correct actions of the technician to help performing the maintenance task more accurate and timely [18].

Furthermore, VibroTac might be a suitable device to apply vibrotactile feedback in rehabilitation situations as presented in [20].

\section{Further ideas}

Besides sensory substitution in virtual reality applications, VibroTac might be used for attention direction in event-driven, information rich environments. As a potential application, the device can be used in air traffic control environments, for instance to direct attention to critical events and/or to maintain situation awareness for aircrafts reaching important waypoints.

Game consoles might become more realistic and their fun factor might be increased if tactile feedback is presented to the player's arms or even to the legs. The device is light and slim and will not disturb the user.

Furthermore, VibroTac might also be used to synchronise motion sequences of several persons for example when dancing or practicing sports.

\section{CONCLUSIONS}

This article introduced an ergonomic vibrotactile feedback device for the human arm. Six vibration segments which can be controlled separately and are continuously adjustable in intensity generate vibrotactile stimuli to the human arm.

As the device is battery powered and has a wireless control, the user profits from natural movement capability. Several devices can be used at the same time since separate control of each device is possible. Thus, a tactile feedback on various parts of the human body can be produced.

Due to the developed concept, this device can be worn on a large range of arm diameters while providing a high level of wearing comfort. Regardless of the different arm diameters, the vibration segments are self-aligning so that they are aligned on the arm in equal distances. The usefulness of this device has been shown in a Virtual Reality automotive assembly verification and a telerobotic system.

Additional vibrotactile feedback to the hand or fingers can be easily realised, as the device possesses a connector with nine additional outputs for vibration units. Depending on the application, a full body vibrotactile feedback would be advantageous. The proposed concept could be used for devices located on other parts of the body e.g. on the legs.

A study to verify the usefulness of VibroTac for attention direction in air traffic control is currently carried out. This study is also used to investigate the reliability of the flexible material of VibroTac when intensively used and to obtain subjective evaluation of ergonomic aspects of the device which can be utilised for future improvements.

\section{ACKNOWLEDGMENTS}

The authors gratefully acknowledge the support of SKILLS Integrated Project (IST-FP6 \#035005, http://www.skills-ip.eu) funded by the European Commission.

\section{REFERENCES}

[1] G. Burdea, Force and touch feedback for virtual reality, A WileyInterscience Publication. Wiley, New York, 1996.

[2] http://www.tactons.org; website checked on July 2010

[3] M. Benali-Khoudja, M. Hafez and J. Alexandre, Tactile interfaces: a state-of-the-art survey, Symposium on Robotics, 2004

[4] R. Baumann, Haptik Interface for Virtual Reality Based Laparoscopic Surgery Training Environment, PhD thesis, Departement de Microtechnique, Polytechnique Federale de Lausanne, 1997.

[5] T. Hulin, M. Sagardia, J. Artigas, S. Schaetzle, P. Kremer and C. Preusche, Human-Scale Bimanual Haptic Interface, Enactive08, Pisa - Italy, 2008

[6] J. B. F. Van Erp, H. A. H. C. Van Veen, C. Jansen and T. Dobbins, Vibrotactile Waypoint Navigation at Sea and in the Air: two Case Studies, ACM Transactions on Applied Perception (TAP); Volume 2 , Issue 2,2005, pp. 106 - $117,2005$.

[7] R. Tadakuma and R.D.Howe, A Whole-Arm Tactile Display System; Proceedings of Worldhaptics, Salt Lake City, USA, 2009.

[8] G. Yang, D. Ryu and S. Kang, Vibrotactile Display for Hand-held Input Device Providing Spatial and Directional Information, Proceedings of Worldhaptics, Salt Lake City, USA, 2009.

[9] P.Lemmens, F. Crompvoets, D. Brokken, J. van den Eerenbeemd and G.-J. de Vries, A body-conforming tactile jacket to enrich movie viewing, Proceedings of Worldhaptics, Salt Lake City, USA, 2009.

[10] D. Tsetserukou and S. Tachi, Efficient Object Exploration and Object Presentation in TeleTA, Teleoperation System with Tactile Feedback, Proceedings of World Haptics, Salt Lake City, USA, 2009.

[11] S. Schaetzle, T. Hulin, C. Preusche and G. Hirzinger, Evalution of Vibro-Tactile Feedback to the Human Arm, Proceedings of Eurohaptics, pp.557-560. Paris, France, 2006.

[12] R.W. Cholewiak and A.A. Collins, Sensory and physiological bases of touch, Lawrence Erlbaum, 1991.

[13] A.M. Murray, R.L. Klatzky and P.K. Khosla, Psychophysical Characterization and Testbed Validation of a Wearable Vibrotactile Glove for Telemanipulation, volume 12. Massachusetts Institute of Technology, April 2003.

[14] M.J. Mueller, Ernaehrungsmedizinische Praxis, Springer Verlag, Germany

[15] T.H. Massie and J.K. Salisbury, The PHANToM Haptic Interface: A Device for Probing Virtual Objects; Proc. of the ASME Winter Annual Meeting, Symposium on Haptic Interfaces for Virtual Environment and Teleoperator Systems, Chicago, USA, Feb 2000.

[16] C. Preusche, A. Rettig and G. Hirzinger, Assembly Verification in Digital Mock-Ups using Force Feedback; 12th International Symposium on Measurement and Control in Robotics Towards Advanced Robot Systems and Virtual Reality, Bourges, France, June 2002.

[17] T. Hulin, C. Preusche and G. Hirzinger, Haptic rendering for virtual assembly verification; Proceedings of World Haptics Conference, Pisa, Italy, March 2005.

[18] S. Webel, T. Engelke, U. Bockholt, F. Tecchia, C. Preusche and N. Gavish, An AR training platform for skills transfer involved in Industrial Maintenance and Assembly Tasks, Research Demonstration at IEEE VR 2010, March 2010.

[19] S. Casado, T. Engelke, N. Gavish, T. Gutiérrez, J. Rodríguez, E.J. Sánches, F. Tecchia, S. Webel, A Training System for Skills Transfer Involved in Industrial Maintenance and Assembly Tasks, SKILLS 09:Enaction on SKILLS, 2009.

[20] P. Kapur, S. Premakumar, S. A. Jax, L. J. Buxbaum, A. M. Dawson, K. J. Kuchenbecker, Vibrotactile feedback system for intuitive upperlimb rehabilitation, Proceedings of World Haptics 2009 - Third Joint EuroHaptics conference and Symposium on Haptic Interfaces for Virtual Environment and Teleoperator Systems, pp.621-622. Salt Lake City, USA, 2009. 